\title{
14 Current challenges in ageing population health intervention research
}

\author{
Illustration with the case of the SoBeezy \\ program
}

\author{
Linda Cambon, François Alla and Karine Pérès
}

\begin{abstract}
In this chapter, we develop issues and methodological aspects of health intervention research in the ageing field. These deal with the complexity of interventions inviting understanding of how these interventions work and can be transferred into another context or scaled up. We illustrate these words with the example of a concrete research project, named SoBeezy.

More precisely, we develop the idea that approaches and research methods have to integrate new research objects, to allow an explicit theoretical hypothesis underlying the levers used within the intervention (designing the theory) and to anticipate the constraints in implementing the innovation into the routine (designing the viability study), with the aim of facilitating the transfer of innovation into practice and to transform organizations.
\end{abstract}

\section{Introduction}

In the context of the rapid ageing of the population, ageing in the best possible conditions has progressively become a major challenge for all societies worldwide. The new demographic transition combined with increasing life expectancies, now realized late in life, raises the issues of health conditions, living conditions, organization of the welfare and health systems and, consequently, of the medical and social costs of ageing.

To face these challenges, we must transform our system and develop and implement innovations, particularly organizational innovations at all levels of care through population health intervention research (PHIR). Developing PHIR in the ageing field raises the following questions: how do we assess these innovative models and interventions considering their complexity, and integrating, at the same time, (i) their efficacy, (ii) the conditions of efficacy and (iii) the conditions of transferability and scalability?

In this chapter, we propose providing methodological insights for PHIR and to illustrate them by a concrete research project, named SoBeezy, in order to answer these questions.

\section{Supporting ageing, an innovation challenge}

In a context of the rapid ageing of the population, ageing in the best possible conditions has progressively become a major challenge for all societies worldwide. According to the World Population Ageing 2017 report, in 2050, two billion people will be aged 60 and older, i.e., more than 21 percent of the world's population, but with great differences by countries (United Nations Department of Economic and Social Affairs, 2017): from around 7 percent in Western Africa countries to more than 40 percent in some European and Asian 
countries (Italy, Greece, Spain, Portugal, Japan, Hong Kong, Taiwan, Republic of Korea and Singapore). The new demographic transition combined with increasing life expectancies, now realized late in life, raises the issues of health conditions, living conditions, organization of welfare and health systems and, consequently, of the medical and social costs of ageing. Indeed, this expected rapid ageing of the population raises concerns about increasing prevalence and incidence of age-related diseases, of chronic illnesses, of polypathology and of all the consequences in terms of dependency and loss of autonomy, both for elderly people and their caregivers.

According to the bio-medical approach of ageing, pathologies are clearly the "gateway" into the ageing process. Prevention is obviously the first level of levers to activate, which is already conducted in classical prevention strategies (with physical activity, diet, tobacco and adequate medical management and care). However, it is now well established that other factors than solely health factors significantly contribute to well-ageing. Defined as contextual factors, they play a significant role in the ageing process (WHO 2019), modulating the impact of disease on people's lives, playing either the role of positive or aggravating factors. Among these potential factors, promising results could be obtained with psychosocial factors; as yet largely underestimated in usual prevention strategies. Among them, combating loneliness and social isolation, and restoring feelings of usefulness to others and self-esteem could be interesting to experiment. Indeed, feelings of loneliness and social isolation have been identified as a real scourge of modern life. With increasing prevalence and incidence over the last decades, they have been recognized as risk factors of poorer quality of life, unhealthy behavior and lifestyle (Shankar et al., 2011; Schrempft et al., 2019; Kharicha et al., 2007), of chronic diseases (depression, anxiety, cardiovascular, Alzheimer's disease) (Valtorta et al., 2016; Grande et al., 2018; Domènech-Abella et al., 2019), dependency (Kharicha et al., 2007) and premature death (Rubin 2017; Tabue Teguo et al., 2016; Tanskanen and Anttila, 2016). In the same way, feelings of uselessness to others, which often appears after the transition to retirement and exacerbates with age, also has a deleterious impact on self-esteem, quality of life, lifestyle, health and even mortality (Gruenewald et al., 2007, 2009; Levy, 2003; Gu, Brown and Qiu, 2016; Okamoto and Tanaka, 2004). Focusing on these levers, an intervention research (IR) approach should be implemented to assess the potential impacts that could be expected on healthy ageing and helping elders staying at home as long as possible in good conditions. Indeed, "ageing in place"1 has clearly become the priority goal of all ageing policies.

Hence, to increase the chance to meet the challenges of ageing populations, we must transform our system and develop and implement innovations, particularly organizational innovations at all levels of care (Alla and Cambon, 2017): to identify research-based and field-based interventions and organizations; to understand what works, how it works, in which context, in which populations and with what impact and effectiveness at both societal and individual level; and to explain the transfer of innovative models into public decisions and practices are the objects of PHIR.

\section{Developing ageing intervention research}

PHIR has been defined as "the science of solutions" (Potvin, Pettcrew and Cohen, 2014), complementary to the science of problems (i.e., study of health determinants). Especially in ageing intervention research (AIR), this calls for several observations. The first is that these solutions are diverse. They could include prevention programs, such as falls prevention programs, or technological innovations, such as a remote fall detection monitoring 
system. They could also refer to organizational innovations as a specific care pathway, or policies such as an elderly plan or social welfare. The second is that PHIR is an operational science, since one of the goals of research is the use of its results by field practitioners and policymakers. This involves taking into account the future transferability and scaling of results in practice and in decision-making (Cambon et al., 2012). This transferability should not be considered post hoc but integrated into the research process itself, challenging perspectives and methods of research (Alla and Cambon, 2017). Indeed, because public health interventions are complex (MRC, 2012), with their results depending on the interaction between the intervention and its context (Cambon, Terral and Alla, 2019), an effective intervention in a given context (especially an experimental one) may not be transferable or scalable.

So, developing PHIR in the ageing field raises the following question: how do we assess these innovative models and interventions considering their complexity, and integrating, at the same time (i) their efficacy, (ii) the conditions of efficacy and (iii) the conditions of transferability and scalability. In this chapter, we propose to provide methodological insights for AIR and to illustrate them by a concrete research project, named SoBeezy, in order to answer these questions.

\section{The SoBeezy project: an innovative program on ageing}

SoBeezy is a large-scale solidarity network based on a digital intelligent platform. Connecting all the resources of a territory, the SoBeezy program aims at facilitating and improving the lives of the most vulnerable people, mainly elderly persons and people living with disabilities. The system proposes solutions to face the main social and material difficulties in activities of daily living and promotes social participation through activities and experience sharing. Whatever the age, gender, socio-professional category, disabilities or diseases, everyone can contribute to the platform, thus restoring feelings of usefulness to others and reducing loneliness. Indeed, beyond the direct benefits expected for the beneficiaries of the services, we assumed that the opportunity given to everyone to contribute to the system allows tackling the sense of uselessness that strikes many elderly people and to give back meaning to life.

In addition to the intergenerational citizen mobilization and contribution, the SoBeezy system relies on all local resources and is organized as a network hub. It includes SoBeezy citizens and different stakeholders of a territory: the municipality and their social services, health and social professionals, other professionals and associations (charities, sports, cultural and leisure associations). This hub design combined with a powerful algorithm guarantees the optimal answer to the expressed needs of the SoBeezy users.

The SoBeezy platform is available on all digital devices: smartphone, tablet and computer. Nevertheless, given the still frequent digital divide in the targeted population, a specific effort has been undertaken to give universal access to the proposed technology. To do so, a specific voice assistant has been developed to facilitate the expression of a need by elderly people, vocally (e.g., "I am looking for someone to ... take me to the "doctor" or "to share a walk" or "to play cards"). In addition, the SoBeezy device also proposes several options to facilitate daily life such as an easy-to use digital calendar, video call, weather, radio, emergency numbers. Thanks to the high usability and accessibility of the device, the SoBeezy voice assistant aims at decreasing apprehensions and fears about technology usage and finally at reducing the digital divide in the elderly population.

Initially designed to meet the specific needs of the elderly population, the SoBeezy program finally also meets other population needs, such as people living with disabilities, 
particularly struck by the scourge of loneliness and feelings of uselessness and also confronted with activity limitations in daily life. To meet the public health and social cohesion challenges of ageing, the SoBeezy program is innovative in many ways:

- Development: co-constructed with elderly users and local stakeholders and partners in charge of the elderly population, the SoBeezy program proposes appropriate solutions to face difficulties and meet the real needs in daily life.

- Levers: SoBeezy targets psychosocial factors less investigated than health factors in usual prevention programs.

- Hypothesis: people who contribute to the platform may have personal benefits in terms of loneliness reduction, reduced feelings of usefulness, gaining meaning out of life, and great encounters and interactions.

- Technology: SoBeezy gives a social utility to technological innovations. With the SoBeezy voice assistant, digitally excluded people could benefit from the network, which is really innovative among the huge amount of technologies currently available.

- Organization: SoBeezy involves and centralizes all the resources of a territory: citizens, professionals and associations.

- Evaluation: a rigorous IR strategy will be conducted to assess the impact and effectiveness of the program on well-ageing.

\section{The effectiveness of AIR}

Hawe and Potvin (2009) described three "myths" of IR, among them: IR is just about intervention effects, is only about controlled trials and controlled interventions. According to them, many different types of study design can actually be used and build evidence but some studies failed because of over-control from researchers on the form of the interventions. Cambon, Terral and Alla (2019) highlight that although public health has historically favored experimental designs because of their internal validity allowing causal attribution, some ethical, technical or legal constraints in PHIR still exist (Tarquinio et al., 2015; Cambon, Terral and Alla, 2019). Some adaptations could be provided to fit with the complexity of interventions. A range of methods could be used successively, or combined at various steps of the evaluation approach (Minary et al., 2019). Among these designs, the most frequently used (Grande et al., 2018) are trial adaptations, quasi-experimental and observational studies, realistic evaluation and other case studies. In short, the aim of the trials' adaptations is to bring them closer to "real life", mainly pragmatic and cluster trials (the latter being also suitable for collective interventions). Quasi-experimental methods (before-and-after design and/or with control group design), observational studies and more generally natural experiments, have the advantage of a stronger external validity than experimental methods, at the cost of a decrease in internal validity. They are sometimes the only ones that can be used (for example, for policy evaluation, which is non-randomizable in nature). Finally, realistic evaluation (Pawson and Tilley, 1997) comes from another scientific paradigm derived from the social sciences. The principle is to explore mechanisms that are activated by the intervention to produce its effects in a given context using mixed methods. Realistic evaluation can provide an explanation on how an intervention functions and in what circumstances (Pawson and Tilley, 1997).

Thus, no "universal" design can be proposed for evaluation of population health intervention, but a range of methods can be used according to the nature of the intervention evaluated and the objective and the context of the evaluation (Grande et al., 2018). 
To illustrate this, in the case of the SoBeezy program, the evaluation protocol has been notably designed to (i) evaluate the use of the device, in terms of acceptability, accessibility, usability and user experience, (ii) assess the impact on well-ageing in terms of feelings of loneliness, sense of usefulness, self-esteem, quality of life, social participation, dependency and healthcare consumption.

The general framework of the research is a prospective pragmatic quasi-experimental study (i.e., an intervention population and a control population with no randomization), involving a cohort of 1000 SoBeezy users recruited in three cities, initially aged 60 years and older, all volunteers to use the platform and to participate to the research. Figure 14.1 illustrates this data collection in steps 1, 2 and 4 (cf. Figure 14.1). The choice of a cluster (i.e., the city) is linked to the collective nature of the intervention, that of a quasiexperimental rather than experimental design, mainly because the preparation and implementation of the intervention required a strong investment from the communities and their representatives. Randomization was not socially acceptable. In addition, the number of voluntary sites was too small and heterogeneous for this randomization to have been effective. Outcomes data (loneliness, sense of usefulness, self-esteem, quality of life, social participation, dependency and healthcare consumption) will be collected at home by a specially trained psychologist at baseline and after 12 months of intervention (step 1 in Figure 14.1). Complementary data will be collected from the SoBeezy device in the ecological context of home and after extraction from the medico-administrative claims datasets. Comparative analyses will be conducted according to different strategies, first, among the SoBeezy users themselves with a before-after design (e.g., in terms of loneliness, feeling of usefulness, dependency). Moreover, two additional comparative analyses will be conducted using specific control groups (step 2 in Figure 14.1). The first, specifically focused on the impact on loneliness, will be conducted comparing two groups of older adults suffering from loneliness: the intervention group of 200 subjects issued from the SoBeezy cohort and the control group including 200 comparable elderly subjects recruited in nonSoBeezy territories. The second approach will consist of a comparison of the short-, mid- and long-term evolution of the SoBeezy-group compared to the general elderly population using the claims data. These two groups will be compared in terms of healthcare utilization (step 4 in Figure 14.1): drugs consumption (with a specific focus on psychotropic drugs), paramedical and medical care use, chronic diseases, hospitalization data, dependency (using a claims-based algorithm) and mortality.

Hence, the efficacy evaluation of SoBeezy was conceived with pragmatic, feasible and rigorous (control groups) approaches in order to allow the implementation and use of the SoBeezy platform during 12 months and the collection of data. Indeed, the SoBeezy project is an alternative design to randomized control trials with a quasi-experimental design embedded in a cohort study.

\section{The conditions of effectiveness of AIR}

In the last 20 years, the number of technological innovations, devices, robots and platforms addressing the issues of assistance, surveillance and prevention for the elderly has dramatically soared. Yet very few of them have been able to prove their effectiveness; some do not meet real needs, others encounter usage-related barriers. But for a large majority of them, conclusions were limited due to the lack of a rigorous intervention research strategy, including processes and mechanisms studies. Such technologies are promising to improve the 


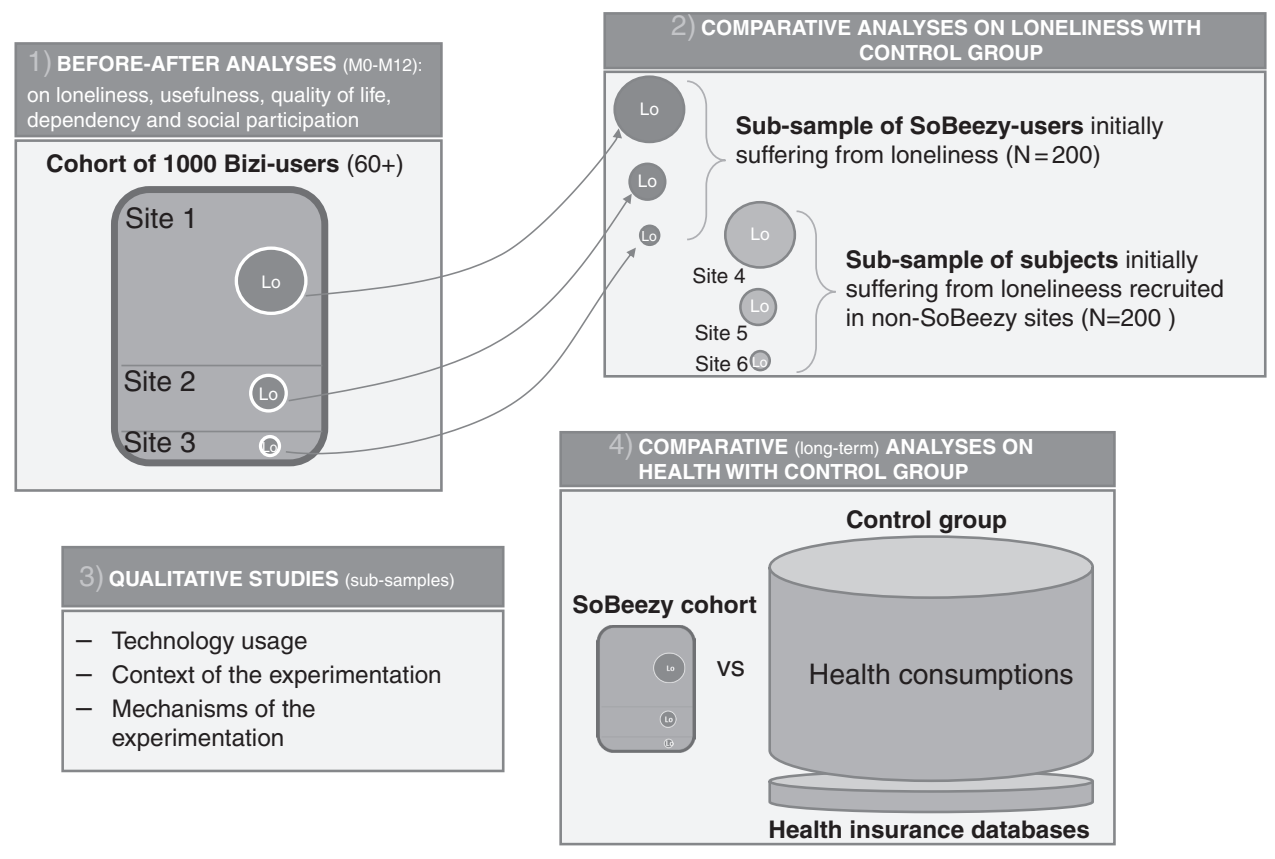

Figure 14.1 Data collection in the SoBeezy program.

well-being of elderly people living at home. However, the lack of high-quality and in-depth studies clearly limits the opportunities of transferability and scaling up (Moore et al., 2015; Marasinghe, Lapitan and Ross, 2015; Chen and Schultz 2016), yet these are crucial for policymakers. Indeed, beyond the feasibility aspects of trials evocated before, efficacy-focused studies (as experimental or quasi-experimental studies) have, if they are used solely, a drawback: they are "blind" to the contextual elements, which influence outcomes. Their potential efficacy may well be demonstrated, but their transferability is weak. And, it becomes a major issue as intervention research is supposed to inform policy and practice (Tarquinio et al., 2015). We talk about the evaluation of a "black box". Yet, the "black box" has to be opened in order to understand how an intervention is effective and how it may be transferred elsewhere. It is about understanding of the conditions of effectiveness. These are the components of intervention, the processes used to implement it, the mechanisms triggered by it and the contextual elements likely influencing the results. Indeed, PHIR, as well as AIR, has to be considered with the complexity perspective of organizational research (as health service research), leading to integrating clearly the context (Hawe, Shiell and Riley, 2004) as coproducing the effects by interactions with interventional components. Some authors as Pawson and Tilley (1997) consider that results in complex intervention are actually derived from configurations between contextual parameters including intervention (C) producing mechanisms $(\mathrm{M})$, which in turn produce outcomes $(\mathrm{O})$. The realistic evaluation framework they have conceived aims to assess these CMO configurations in order to open the black box and answer the following question: for whom and in which conditions does the intervention work? In this respect, Cambon, Terral and Alla (2019) have argued that another major myth of PHIR is the willingness to distinguish arbitrarily intervention from context. They advocate 
that the complexity has to be assumed and propose a new definition of the object of public health studies - the "interventional system" - which includes interventional, contextual components (Shiell, Hawe and Gold, 2008) and the mechanisms triggered, these last considered as the key elements of this interventional system.

In this way, another challenge in AIR could be the understanding of these mechanisms and the ways they are produced. It involves tracking (in-itinere) in process evaluation the mechanisms as they are hypothesized and as they emerge, expected or not, during implementation. It leads to rethinking the evaluation approach and methods, exploring the interventional system as different from the sum of its (interventional or contextual) parts by a contributive analysis (Mayne, 2001). Process evaluation is the point of any guidelines for helping the researcher to take into account these questions and consider this complexity (Moore et al., 2015; Craig et al., 2018). One way to assess process evaluation is theorydriven evaluations (Alla and Cambon, 2017), solely performed or complementary with trials, because they use this contributive understanding of an intervention through mechanistic interpretation focusing on the exploration of causal chains. In theory-driven evaluations, the causal combinations of components are hypothesized by combining scientific evidence and the expertise of practitioners and researchers. The hypothetical system is then tested empirically. If this is conclusive, evidence therefore exists of contribution, and causal inferences can be made. Two main categories of theory-driven evaluations can be distinguished: realist evaluation and theories of change (Blamey and Mackenzie, 2007). In both cases and in an interventional system perspective, context and interventional components have to be equally considered in the analyses.

In the case of SoBeezy study, the understanding of mechanisms is the reason why a third objective has been added: analyze the intervention processes, context and mechanisms that lead to the results obtained. Indeed, the SoBeezy program is a complex intervention, which can be highly influenced by contextual elements. The SoBeezy system relies on different actors (citizens, professionals and associations), concerned directly or indirectly with the elderly population. This multiplicity of partners, which is one of the main strengths of the program, also represents a complexity to evaluate. The efficacy of the system will largely depend on the level of involvement of the different partners; a level which can vary between territories according to local policies, to the dynamism of the associational network, to the involvement of professionals and to the sweep of citizen mobilization. Moreover, other contextual elements can also influence the results. Thus, the choice has been made to experiment with SoBeezy in three pilot territories (three cities of southwestern France) very different in terms of number of inhabitants (from 6800 to 62,000), proportion of elderly people ( 22 percent to 41 percent), population density (67 to 1580 inhabitants $/ \mathrm{km}^{2}$ ), urban or rural areas, medical and paramedical demography, access to transport, and services and digital coverage. All these parameters may influence the obtained results of the intervention. Moreover, the general objective of the SoBeezy program is to favor well-ageing and ageing at home in good conditions. To do so, five mechanisms are targeted by the program, and hence studied (cf. Figure 14.2).

- Loneliness and social isolation. With more than 20 percent of the elderly population affected by loneliness and, given its deleterious impact on quality of life and health, the SoBeezy program first proposes visits at home by citizens or trained volunteers from associations already involved in the battle against loneliness. Another less direct way to tackle loneliness, probably less stigmatizing, is the experiences and activities sharing component of the SoBeezy program. Finally, being personally involved in the 


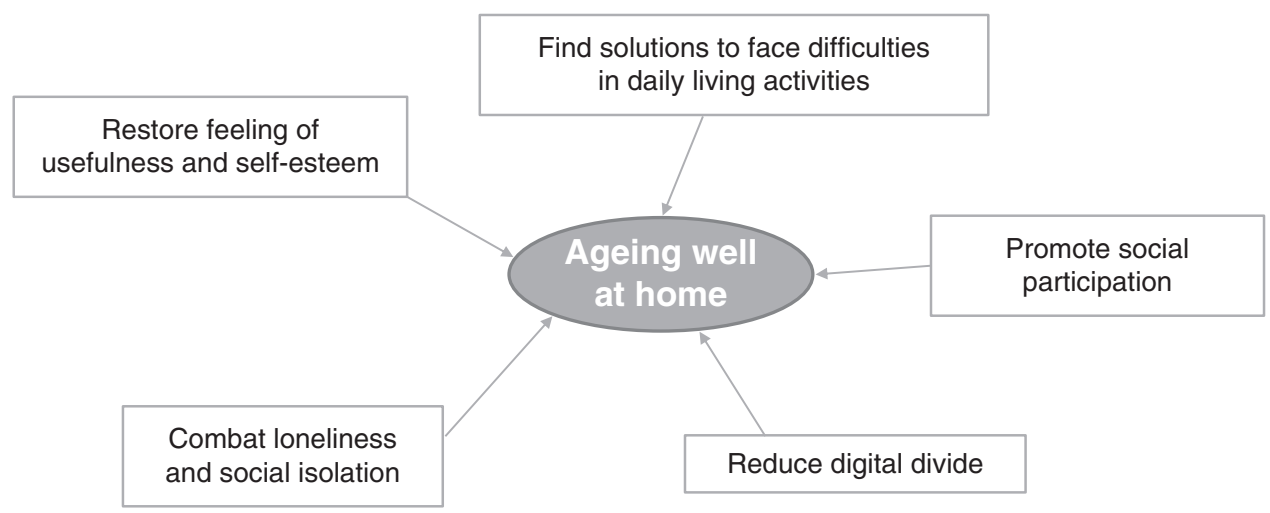

Figure 14.2 SoBeezy mechanisms of effect.

system for other people and belonging to the SoBeezy community should also contribute to reducing loneliness and isolation.

- Feelings of usefulness and self-esteem. With ageing, the retirement period (sometimes associated with loss of social status and "daily life routine destructuring"), loss of spouse, relatives and friends, diseases and disabilities etc., feelings of uselessness and loss of self-esteem often occur and worsen with age. The SoBeezy program gives the opportunity to everyone to get involved for other people, as a helping hand, a sympathetic ear and an encouraging and caring smile for persons struggling with loneliness, dependency and illness.

- Facing difficulties in activities of daily living. Such limitations can jeopardize the chance of staying at home, especially for people living alone. Thanks to local large resources referenced and centralized in the SoBeezy Hub, the platform will be able to identify the optimal answer to meet the needs of the SoBeezy users. Based on previous studies, the services proposed by the system should cover the main needs of the elderly people: transportation, shopping, punctual aid for housekeeping and gardening, digital training, assistance for administrative tasks (mainly now digitalized) and pet sitting and care. Some services will be either provided by citizens and volunteers (freely), or by professionals (paid) when specific skills are required or when no free solutions are found by the platform.

- Social participation. In the last years of life, most of us are concerned by diseases and disabilities. On this basis, instead of considering successful ageing as the absence of diseases, well-ageing could be considered as living with preserved social participation despite disease and functional limitations. This goal will rely on all the local actors and partners of the territory (municipality and associations), as well as on all the social activities proposed by the SoBeezy community.

- Digital divide. All administrative tasks are becoming digitalized at a steady pace. Consequently, being digitally excluded starts to become a real social disadvantage. Given the high frequency of digital divide in the current generation of elderly people, the SoBeezy system provides volunteer digital trainers and easy-to use technological devices to reduce fear, apprehension, aversion to technologies and to give universal access to technologies and to the Internet. 
To analyze both contextual elements and mechanisms, we will perform a realistic evaluation embedded in the efficacy design. Concretely, qualitative studies to analyze technology usage, the context and the mechanisms of the experimentation have been added to the efficacy study described above. This qualitative investigation will be conducted through non-structured and semi-structured interviews with all stakeholders and older people involved in the intervention group. The aim is to figure out the combinations of recurrent CMOs in the intervention group in the three towns in order to answer these questions: (i) Through which mechanisms does the SoBeezy system produce some results (M-O)? (ii) What key elements of the SoBeezy system are the most effective (C-O)? (ii) What key elements of the implementation of the SoBeezy system are the most effective (C-O)? (iv) What are the factors that depend on people's and environments' characteristics which influence the mechanisms triggered and the effects (C-M-O)? This data collection is illustrated, complementary to the others in Figure 14.1 in step 2 (cf. Figure 14.1).

Hence, complementary to the efficacy evaluation, this complementary study will contribute to understand in which conditions the SoBeezy platform is effective: which components of the interventional system - population and stakeholders, implementation, external, internal to the platform characteristics - and through which mechanisms are they effective?

\section{Considering transferability and scalability in AIR}

Despite this methodological development, PHIR remains undersized and the findings are not always used by decision-makers and practitioners for many reasons. One of them is that the knowledge produced is not always adapted or usable because it does not take adequate account of real-world circumstances. To avoid this, the production of knowledge has to be made with an interacting framework, including all stakeholders and their points of view, in order to verify that the intervention can be routinized in a real-life context. We talk about viability analysis (Chen, 2010), which has been considered an essential prerequisite in PHIR, and hence in AIR. Indeed, in a perspective of use, what is the point of analyzing the effectiveness of an intervention in an experimental context that is not a real-life one without first ensuring that if it is effective, it will be transferable in real life? Chen (2010) recommends addressing the "viability validity" of an intervention as the first step in an evaluation process. He has defined this concept as "the extent to which an evaluation provides evidence that an intervention is successful in the real world", referring to the practical, affordable, suitable, evaluable and helpful aspects of an intervention in the real world. In other terms, it refers to the way an intervention fits with the context, especially answering stakeholders' needs and interests. According to Chen, without a viability assessment, we risk addressing the efficacy of an "off ground" intervention that cannot be transferred to another context or scaled up. The proposal is to use an integrative validity approach, first proceeding to a viability study, second performing an effectiveness study considering the real conditions of implementation and last (and if necessary) conducting an efficacy study or directly proceeding to dissemination. Practically, if the intervention is research based, this viability analysis should be integrated into the objectives of a pilot study in addition to its classical objectives (Thabane et al., 2019) in research-design program evaluation in order to prepare as best as possible both trial and intervention by reporting essential information to transfer or scale up the intervention. If the intervention is field based, it is by nature 
adapted to the context, conceived with local and routine resources. This kind of intervention is likely more transferrable than the others. We talk about natural experiment designs (Craig et al., 2012); because the intervention is not conceived and implement by researchers, the research is added second to evaluate the ongoing field intervention. In this case the viability study aims to understand the possible adaptations of the "intervention prototype" by assessing its "key functions" (Hawe, Shiell and Riley, 2004). The key functions are basic elements considered as key to producing an effect, for example an intervention's theoretical processes ("key functions") which have to be distinguished from structural and contingent parameters (Hawe, Shiell and Riley, 2004). In both cases, research- or field-based interventions, the aim is to understand how and under what conditions intervention components could fit with routine conditions, answer different stakeholders needs and be able to be adapted in another context or resources scale up.

The SoBeezy program is the second case evocated: a field-based program. But, given the complexity of the SoBeezy organization and the potentially important influence of the contextual factors (due to the heterogeneity between territories in terms of needs, dynamism and mobilization of each partner), the transferability issue remains a major point. It is the reason why (i) technological and organizational pre-tests have been made with 35 elderly users living at home and in retirement homes, (ii) they have been tested in real conditions of use, (iii) they have been implemented at a city scale. The whole city is potentially exposed to the SoBeezy program and each person (citizen, volunteer or professional) represents a potential resource to the system. Thanks to the diversity of the three pilot sites, a specific analysis of the special influence of the contextual factors on the impact and efficacy of the intervention will be conducted, which will provide crucial data in a perspective of transferability to another context and scaling up. Data will be collected by the same interviews performed in realistic evaluation, mentioned above, in order to answer these both questions: What are the elements to be considered when transferring this intervention in other contexts: preparatory modalities, resources, types of professionals involved, culture, etc.? Which adaptations have been made in the implementation in the three contexts in terms of resources, costs, modalities of implementation? This viability assessment aims to provide essential data to consider the relevance of efficacy evaluation. Indeed, an intervention could be effective but unfeasible or unwilling for cultural, ethical reasons for example. Conducted in a decision perspective, it provides information about what is really feasible, adaptable and scalable in the SoBeezy concept. Hence, the viability study, imbedded in the realistic evaluation, becomes a complement to the efficacy evaluation. Mechanisms, process and viability data collection contribute to provide some recommendations to implement and scale up SoBeezy. This involves a global and complete approach of ageing, a multidisciplinary strategy will be implemented, with both quantitative and qualitative approaches and several disciplines represented: epidemiology, public health, psychology, sociology, cognitics, economy, computer science and statistics. Admittedly, the research becomes more complex! But it also becomes a source of more learning in a perspective of decision and change of practices. It fits more the complexity of the intervention itself.

\section{Conclusion}

To conclude, PHIR, and especially AIR, is a question of perspectives, moving concepts, approaches and research methods by the integration of new concepts such as context, 
mechanisms, viability and theory. This means doing research differently, based on interdisciplinarity and involving the different stakeholders in order (i) to make explicit the theoretical hypothesis underlying the levers used within the intervention (designing the theory), (ii) to anticipate the constraints in implementing the innovation into routine (designing the viability study) with the aim of facilitating the transfer of innovation into practice and transform the organizations. Considering, as such, the complexity of ageing intervention could contribute to change and improve the welfare and health systems to face the current challenges arising from the ageing of the population.

\section{Note}

1 "Ageing in place" is defined by the Centers for Disease Control and Prevention as "the ability to live in one's own home and community safely, independently, and comfortably, regardless of age, income, or ability level" (www.cdc.gov).

\section{References}

Alla, F. and Cambon, L. (2017). Transformation of health systems: contribution of population health intervention research. Lancet Public Health, 2(12), e539.

Blamey, A. and Mackenzie, M. (2007). Theories of change and realistic evaluation. Evaluation, 13, 439-455.

Cambon, L., Minary, L., Ridde, V. and Alla, F. (2012). Transferability of interventions in health education: a review. BMC Public Health, 12, 497.

Cambon, L., Terral, P. and Alla, F. (2019). From intervention to interventional system: towards greater theorization in population health intervention research. BMC Public Health, 19(1), 339.

Chen, H.T. (1990). Theory-driven evaluation. Thousand Oaks, CA: Sage.

Chen, H.T. (2010). The bottom-up approach to integrative validity: a new perspective for program evaluation. Evaluation and Program Planning, 33, 205-214.

Chen, Y-R.R. and Schulz, P.J. (2016). The effect of information communication technology interventions on reducing social isolation in the elderly: a systematic review. Journal of Medical Internet Research, 18(1), e18.

Craig, P., Cooper, C., Gunnell, D., Haw, S., Lawson, K., Macintyre, S., Ogilvie, D., Petticrew, M., Reeves, B., Sutton, M. and Thompson, S. (2012). Using natural experiments to evaluate population health interventions: new MRC guidance. Journal of Epidemiology and Community Health, 66(12), 1182-1186.

Craig, P., Ruggiero, E.D., Frohlich, K.L., Mykhalovskiy, E., White, M., on behalf of the Canadian Institutes of Health Research (CIHR)-National Institute for Health Research (NIHR) Context Guidance Authors Group (2018). Taking account of context in population health intervention research: guidance for producers, users and funders of research. NIHR Journals Library. doi: 10.3310/CIHR-NIHR-01.

Domènech-Abella, J., Mundó, J., Haro, J.M. and Rubio-Valera, M. (2019). Anxiety, depression, loneliness and social network in the elderly: Longitudinal associations from The Irish Longitudinal Study on Ageing (TILDA). Journal of Affective Disorders, 246, 82-88.

Grande, G., Vetrano, D.L., Cova, I., Pomati, S., Mattavelli, D., Maggiore, L., Cucumo, V., Ghiretti, R., Vanacore, N., Mariani, C. and Rizzuto, D. (2018). Living alone and dementia incidence: a clinical-based study in people with mild cognitive impairment. Journal of Geriatric Psychiatry and Neurology, 31(3), 107-113.

Gruenewald, T.L., Karlamangla, A.S., Greendale, G.A., Singer, B.H. and Seeman, T.E. (2007). Feelings of usefulness to others, disability, and mortality in older adults: the MacArthur study of successful ageing. Journal of Gerontology: Series B, 62(1), 28-37. 
Gruenewald, T.L., Karlamangla, A.S., Greendale, G.A., Singer, B.H. and Seeman, T.E. (2009). Increased mortality risk in older adults with persistently low or declining feelings of usefulness to others. Journal of Ageing and Health, 21(2), 398-425.

Gu, D., Brown, B.L. and Qiu, L. (2016). Self-perceived uselessness is associated with lower likelihood of successful ageing among older adults in China. BMC Geriatrics, 16(1), 172.

Hawe, P. and Potvin, L. (2009). What is population health intervention research? Canadian Journal of Public Health. Revue canadienne de santé publique, 100(1), Suppl. I8-14.

Hawe, P., Shiell, A. and Riley, T. (2004). Complex interventions: how "out of control" can a randomised controlled trial be? BMJ Australia, 328, 1561-1563.

Kharicha, K., Iliffe, S., Harari, D., Swift, C., Gillmann, G. and Stuck, A.E. (2007). Health risk appraisal in older people 1: are older people living alone an "at-risk" group? British Journal of General Practice, 57(537), 271-276.

Levy, B.R. (2003). Mind matters: cognitive and physical effects of ageing self-stereotypes. Journal of Gerontology: Series B, 58(4), P203-211.

Marasinghe, K.M., Lapitan, J.M. and Ross, A. (2015). Assistive technologies for ageing populations in six low-income and middle-income countries: a systematic review. BMJ Innovations, 1(4), $182-195$.

Mayne, J. (2001). Addressing attribution through contribution analysis: using performance measures sensibly. Canadian Journal of Program Evaluation, 16(1), 1-24.

Minary, L., Trompette, J., Kivits, J., Cambon, L., Tarquinio, C. and Alla, F. (2019). Which design to evaluate complex interventions? Toward a methodological framework through a systematic review. BMC Medical Research Methodology, 19(1), 92.

Moore, G.F., Audrey, S., Barker, M., Bond, L., Bonell, C., Hardeman, W., Moore, L., O'Cathain, A., Tinati, T., Wight, D. and Baird, J. (2015). Process evaluation of complex interventions: Medical Research Council guidance. $B M J, 350$, h1258.

MRC (ed.) (2012). Developing and evaluating complex interventions: new guidance. London: Medical Research Council.

Okamoto, K. and Tanaka, Y. (2004). Subjective usefulness and 6-year mortality risks among elderly persons in Japan. Journal of Gerontology: Series B, 59(5), P246-249.

Pawson, R. and Tilley, N. (1997). Realistic evaluation. Thousand Oaks, CA: Sage.

Potvin, L., Petticrew, M. and Cohen, E.R.M. (2014). Population health intervention research: developing a much needed science of solutions. Preventive Medicine, 61, 114-115.

Rubin, R. (2017). Loneliness might be a killer, but what's the best way to protect against it? JAMA, 318(19), 1853-1855.

Schrempft, S., Jackowska, M., Hamer, M. and Steptoe, A. (2019). Associations between social isolation, loneliness, and objective physical activity in older men and women. BMC Public Health, 19(1), 74.

Shankar, A., McMunn, A., Banks, J. and Steptoe, A. (2011). Loneliness, social isolation, and behavioral and biological health indicators in older adults. Health Psychology, 30(4), 377-385.

Shiell, A., Hawe, P. and Gold, L. (2008). Complex interventions or complex systems? Implications for health economic evaluation. BMJ, 336(7656), 1281-1283.

Tabue Teguo, M., Simo-Tabue, N., Stoykova, R., Meillon, C., Cogne, M., Amiéva, H., and Dartigues, J.F. (2016). Feelings of loneliness and living alone as predictors of mortality in the elderly: the PAQUID study. Psychosomatic Medicine, 78(8), 904-909.

Tanskanen, J. and Anttila, T. (2016). A prospective study of social isolation, loneliness, and mortality in Finland. American Journal of Public Health, 106(11), 2042-2048.

Tarquinio, C., Kivits, J., Minary, L., Coste, J. and Alla, F. (2015). Evaluating complex interventions: perspectives and issues for health behaviour change interventions. Psychology and Health, 30, $35-51$.

Thabane, L., Cambon, L., Potvin, L., Pommier, J., Kivits, J., Minary, L., Nour, K., Blaise, P., Charlesworth, J. and Alla, F. (2019). Population health intervention research: what is the place for pilot studies? Trials, 20(1), 309. 
Valtorta, N.K, Kanaan, M., Gilbody, S., Ronzi, S. and Hanratty, B. (2016). Loneliness and social isolation as risk factors for coronary heart disease and stroke: systematic review and meta-analysis of longitudinal observational studies. Heart, 102(13), 1009-1016.

WHO (2019). International Classification of Functioning, Disability and Health (ICF). World Health Organization. Available at: www.who.int/classifications/icf/en/ [Accessed 29 August 2019].

United Nations Department of Economic and Social Affairs (2017). World population ageing 2017. New York: United Nations. Available at: www.un.org/en/development/desa/population/theme/ ageing/WPA2017.asp [Accessed 29 August 2019].

Weiss, C.H. (1997). How can theory-based evaluation make greater headway? Evaluation Review, 21(4), 501-524. 